\title{
Radiological-pathological correlation of pleomorphic liposarcoma of the anterior mediastinum in a 17-year-old girl
}

\author{
Maysa Saeed • Sara Plett • Grace E. Kim • \\ H. Daldrup-Link • J. Courtier
}

Received: 9 February 2010 /Revised: 30 June 2010 / Accepted: 13 July 2010 /Published online: 9 September 2010

(C) The Author(s) 2010. This article is published with open access at Springerlink.com

\begin{abstract}
Liposarcoma is a soft-tissue sarcoma typically seen in adults. It is extremely rare in children. It most often occurs in the extremities or in the retroperitoneum. We present a very rare case of an anterior mediastinal liposarcoma of the pleomorphic subtype in a 17-year-old girl, along with radiological and pathological correlation. The location, patient age and histological subtype are exceedingly uncommon for this tumor.
\end{abstract}

Keywords Liposarcoma · Anterior mediastinum · Child . Surgery

\section{Introduction}

Liposarcoma is a soft-tissue sarcoma that is extremely rare in children. It is classified as a mesenchymal malignant neoplasm with fatty tissue differentiation. Liposarcomas are

\author{
M. Saeed $(\bowtie)$ \\ Undergraduate, University of California, Santa Cruz, \\ 70 Pinto Lane, \\ Novato, CA 94947, USA \\ e-mail: maysa08@aol.com \\ G. E. Kim \\ Department of Pathology, University of California San Francisco, \\ San Francisco, CA, USA \\ S. Plett $\cdot$ H. Daldrup-Link $\cdot$ J. Courtier \\ Department of Radiology, University of California San Francisco, \\ San Francisco, CA, USA \\ Present Address: \\ S. Plett \\ Department of Radiology, \\ Columbia University College of Physicians and Surgeons, \\ New York, NY, USA

commonly found in the retroperitoneum and the extremities. We report a case of a large $(20 \mathrm{~cm} \times 8 \mathrm{~cm})$ liposarcoma localized in the mediastinum of a 17-year-old girl. This tumor was treated surgically with resection, followed by adjuvant chemotherapy and radiation therapy.

\section{Case report}

A 17-year-old girl was referred by her primary care physician because of an abnormality on an echocardiogram from another hospital. The echocardiogram reported a $20-\mathrm{cm}$ anterior mediastinal mass.

The girl's symptoms prompting the echocardiogram were shortness of breath, chest discomfort and an unusual heart murmur. She also reported fatigue and a 6-lb. weight loss. She did not experience night sweats, fevers or chills. In light of the echo results and clinical presentation, the patient was referred to pediatric oncology. A follow-up chest CT scan revealed a $20 \mathrm{~cm} \times 8 \mathrm{~cm}$ heterogeneous tumor with various areas of soft-tissue density and elements of fat tissue (Fig. 1). Her left main stem bronchus was compressed by the mass. Increased displacement of the vessels was noted.

Over the next month, the girl's chest discomfort worsened. She underwent surgery for resection of the mass. The irregular, ovoid $22 \times 16 \times 5 \mathrm{~cm}$ mass weighed $941 \mathrm{~g}$ and had a solid, but lobulated, yellow-orange to pink cut surface (Fig. 2).

Microscopically, the neoplastic cells ranged from small to larger polygonal cells with abundant eosinophilic granular cytoplasm, many of which had variably prominent cytoplasmic vacuoles. They were arranged in sheets of trabeculae and nests (Fig. 2). These epithelioid-appearing cells had multiple small vacuoles dispersed within the granular cytoplasm in some areas, while other areas had up to a few large vacuoles pushing the nucleus to the side 

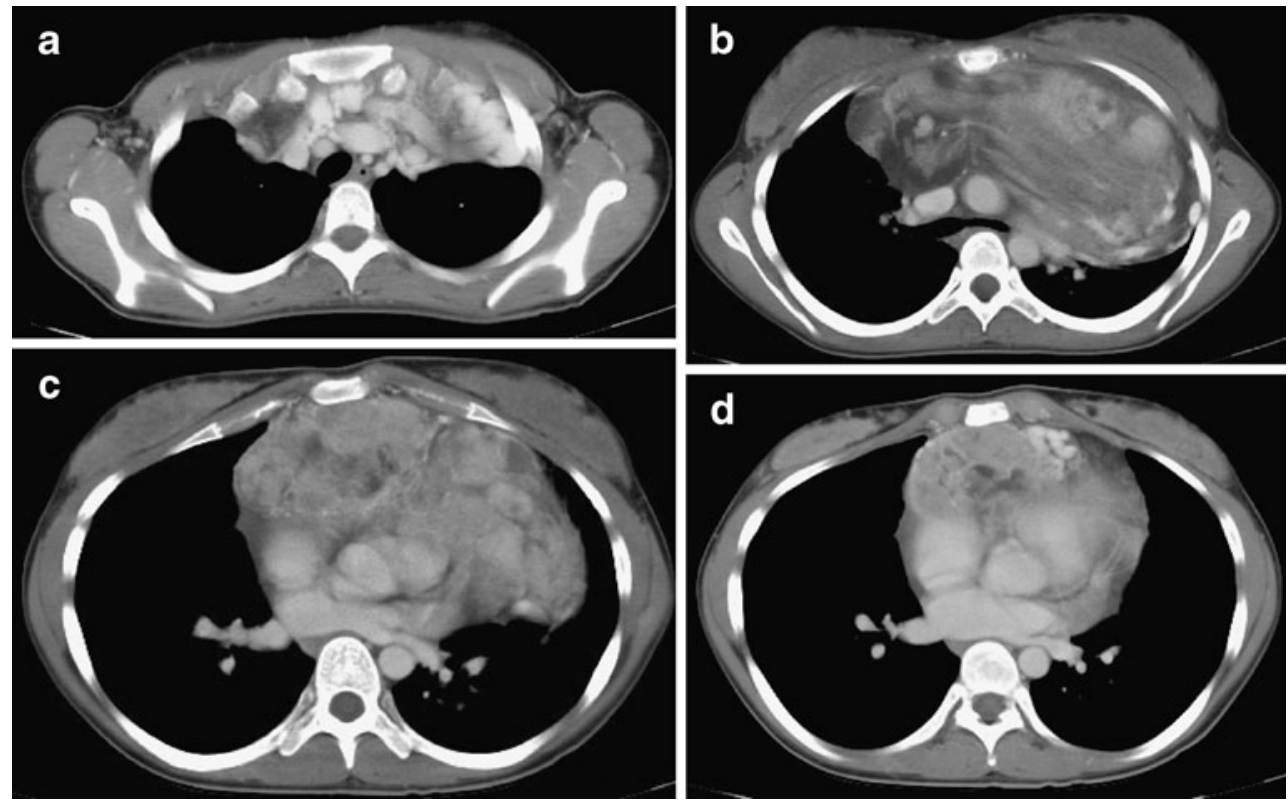

Fig. 1 Chest CT. a An axial image of the chest status after administration of IV contrast (2 cc/ $\mathrm{kg}$ Omnipaque 350, GE Healthcare Inc., Princeton, N.J.) at the level of the superior mediastinum demonstrates multiple enlarged, tortuous collateral vessels. b Level of the carina demonstrating an enlarged heterogeneous mass with areas of fat attenuation in the anterior mediastinum. The mediastinal

contents are displaced posteriorly. No mediastinal lymphadenopathy is present. c A scan at the level of the superior pulmonary veins demonstrates a more extensive soft-tissue component of the anterior mediastinal mass. d A scan at the level of the inferior pulmonary veins demonstrates the inferior margin of the anterior mediastinal mass
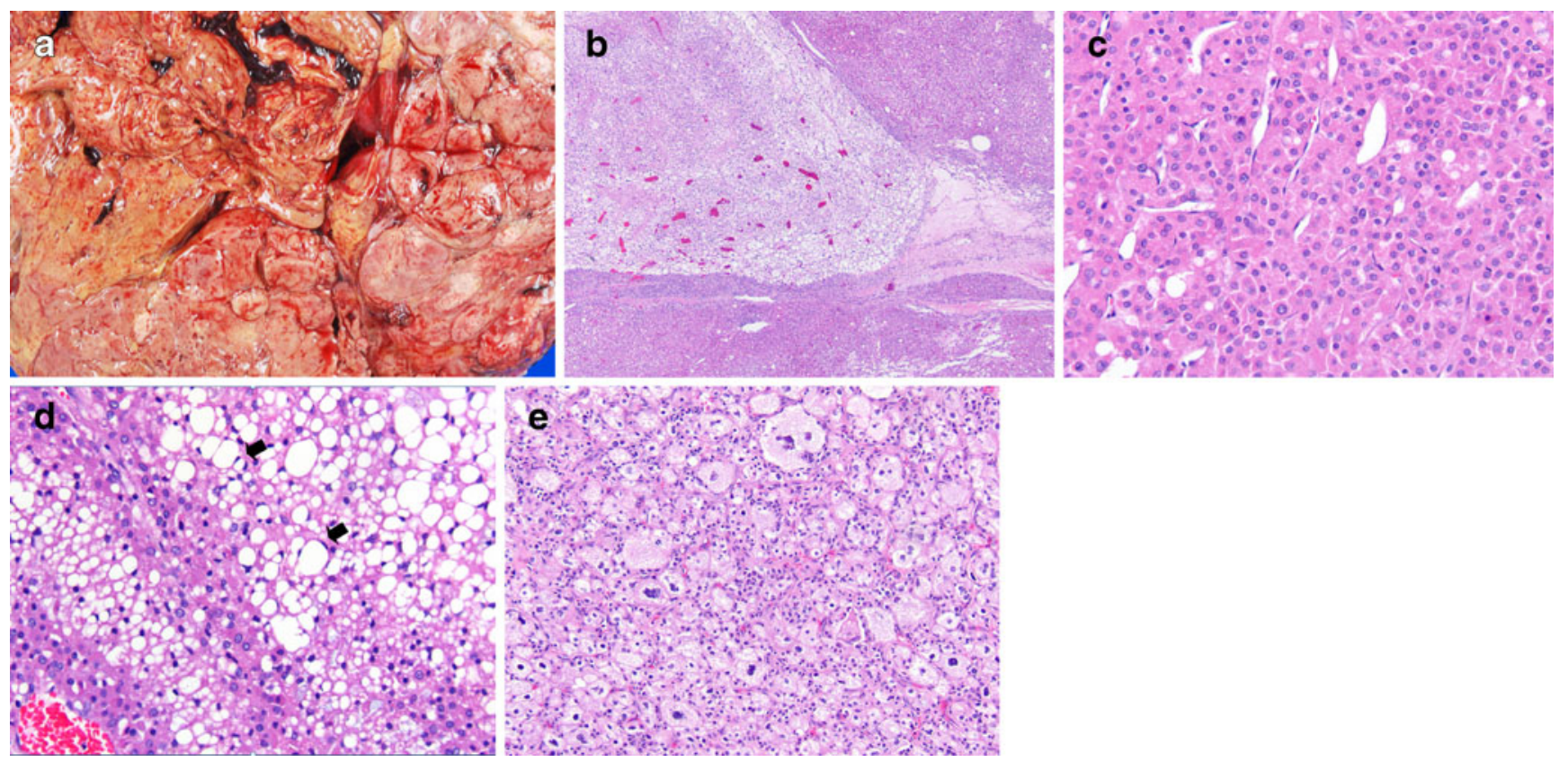

Fig. 2 Histology. a The gross appearance of the epithelioid variant of pleomorphic liposarcoma depicts a portion of the large $(22 \times 16 \times 5 \mathrm{~cm})$, solid, multilobated mass with yellow-orange and pink-white areas. b Hematoxylin and eosin stain: Low magnification shows most of the tumor cells had abundant granular eosinophilic cytoplasm (right upper and lower pink areas), but focal zones of the tumor also had varying amounts of cytoplasmic lipid (central to left pink white area) confirmed with an oil red $\mathrm{O}$ stain (not shown). c
Hematoxylin and eosin stain: This higher magnification shows the trabecular arrangement of the granular epithelioid tumor cells with distinct cytoplasmic borders separated by capillaries resembling an epithelioid neoplasm. d Hematoxylin and eosin stain: This higher magnification reveals prominent cytoplasmic vacuolation, including signet ring-like lipoblasts (black arrows), but convincing multivacuolated lipoblasts were not identified. e Hematoxylin and eosin stain: Only focal nuclear pleomorphism was present 
(signet ring-like lipoblasts, Fig. 2). In one distinct focus, the cells had larger hyperchromatic pleomorphic nuclei with abundant finely granular cytoplasm without vacuolated cytoplasm (Fig. 2).

The morphological features were reminiscent of an epithelial tumor, such as adrenal cortical carcinoma, or a hepatocellular neoplasm with fatty change. The differential diagnosis included epithelial neoplasms and other tumors, such as hepatoid yolk sac tumor, perivascular epithelioid cell tumor and the epithelioid variant of pleomorphic liposarcoma. A histochemical stain for oil red O confirmed that the vacuoles contained lipid (not shown). Immunohistochemical stains were negative for synaptophysin, chromogranin, inhibin and Melan A, excluding a neuroendocrine tumor or adrenal cortical carcinoma. Despite the hepatoid appearance, the mass was not reactive for HepParl, glypican-3 and AFP and, therefore, unlikely to be a a hepatoid variant of yolk sac tumor. These as well as other carcinomas were further excluded by the negative staining for pancytokeratin and EMA. Negative stain for HMB45 provided no support for a perivascular epithelioid cell tumor. Other negative stains included S100 protein, SMA, desmin, caldesmon, myogenin, CDK4, CD34 and CD31. An epithelioid variant of pleomorphic liposarcoma was diagnosed based on the morphological, histochemical and immunohistochemical findings.

Surgery was followed by adjuvant chemotherapy with doxorubicin (total dose $375 \mathrm{mg} / \mathrm{m} 2$ ) and ifosfomide (total dose $54 \mathrm{mg} / \mathrm{m}^{2}$ ) as well as radiation therapy (total dose $55.8 \mathrm{~Gy})$ to the anterior mediastinum.

\section{Discussion}

Liposarcoma is a rare soft-tissue malignant tumor derived from primitive embryonic tissues. It represents approximately $1 \%$ of all soft-tissue malignancies in adults. Most commonly, these tumors are found in the lower extremities; mediastinal involvement is exceedingly rare, with fewer than 150 cases reported in the literature [1]. Typically, liposarcomas present in adults in their $50 \mathrm{~s}$ or $60 \mathrm{~s}$; patients often remain asymptomatic until the tumor reaches a critical size. Patients with mediastinal involvement clinically present with chest pain, dyspnea and possible superior vena cava syndrome. There may also be fatigue and weight loss. Interestingly, rare cases of mediastinal liposarcoma in children as young as 12 years old have also been reported [2].

Histologically, liposarcoma is divided into three major subtypes: well-differentiated myxoid/round cell, dedifferentiated and pleomorphic [3]. Myxoid liposarcoma reportedly accounts for $40-50 \%$ of these tumors [4]. These histological subtypes correlate with potential for metastasis; well-differentiated liposarcomas are considered to be the least aggressive, while myxoid liposarcomas have been reported to disseminate to pleural, pericardial and diaphragmatic surfaces [5].

The histology of this case was specifically of the pleomorphic type, epithelioid variant. It is the rarest of the subtypes and its recognition requires careful histopathological examination for pleomorphic lipoblasts for accurate diagnosis [6]; however, in the original description of this tumor Miettinen et al. describes that the lipoblastic differentiation was most often of the signet ring type [7].

Pleomorphic liposarcoma was first described in adult patients and is considered a high-grade sarcoma with significant risk of metastasis [7]. The primary locations were the extremities and chest wall.

Typical imaging findings of mediastinal liposarcoma include mediastinal widening on chest radiography with possible tracheal deviation. Depending on the degree of differentiation, mediastinal liposarcoma may appear on CT or MR as a fatty mass with a variable soft-tissue component. The solid components may enhance and there may be infiltration or displacement of adjacent structures [8].

The classical treatment for mediastinal liposarcoma is surgical resection, often with adjuvant chemotherapy and radiation therapy. Same-site recurrence following surgery is common, often due to an incomplete surgical resection, thus adjuvant therapy is recommended.

In conclusion, we document a case of a primary anterior mediastinal pleomorphic liposarcoma in a 17-year-old girl. To our knowledge, the radiological appearance of this rare subtype of liposarcoma is unique.

Open Access This article is distributed under the terms of the Creative Commons Attribution Noncommercial License, which permits any noncommercial use, distribution and reproduction in any medium, provided the original author(s) and source are credited.

\section{References}

1. Barbetakis N, Samanidis G, Samanidou EK et al (2007) Primary mediastinal liposarcoma:a case report. J Med Case Rep 1:161

2. Hirai S, Hamanaka Y, Mitsui N et al (2007) Surgical resection of primary liposarcoma of the anterior mediastinum. Ann Thorac Cardiovasc Surg 14:38-41

3. Fletcher CD, Unni KK (eds) (2002) Pathology and genetics: tumours of soft tissue and bone. World Health Organization Classification of Tumours. IARC Press, Lyon, pp 35-46

4. Shoji T, Sonobe M, Okubo K et al (2009) Giant primary liposarcoma of the chest. Gen Thorac Caridiovasc Surg 57:159-161

5. McLean TR, Almassi GH, Hackbarth DA et al (1989) Mediastinal involvement by myxoid liposarcoma. Ann Thorac Surg 47:920-921

6. Downes KA, Goldblum JR, Montgomery EA et al (2001) Pleomorphic liposarcoma: a clinicopathologic analysis of 19 cases. Mod Pathol 14:179-184

7. Miettinen M, Enzinger FM (1999) Epithelioid variant of pleomorphic liposarcoma: a study of 12 cases of a distinctive variant of high-grade liposarcoma. Mod Pathol 12:722-728

8. Munden RF, Nesbitt JC, Kemp BL et al (2000) Primary liposarcoma of the mediastinum. AJR 175:1340 